\title{
Successful treatment of refractory adult onset Still's disease with rituximab
}

\author{
N. Belfeki, M. Smiti Khanfir, F. Said, A. Hamzaoui, T. Ben Salem, \\ I. Ben Ghorbel, M. Lamloum, M.H. Houman \\ Department of Internal Medicine, University Hospital of La Rabta, Tunis, Tunisia
}

\begin{abstract}
SUMMARY
Adult-onset Still's disease (AOSD) is an uncommon inflammatory condition of unknown origin. In chronic disease, joint involvement is often predominant and erosions are noted in one third of patients. Therapeutic strategies derive from observational data. Corticosteroids are usually the first-line treatment. With inadequate response to corticosteroids, methotrexate appears the best choice to control disease activity and allow for tapering of steroid use. For refractory disease, biological therapy seems the most promising. We report here the case of a 38-year-old female patient with AOSD refractory to cytotoxic agents, treated by rituximab infusion therapy with favorable outcome.
\end{abstract}

Key words: Adult-onset Still's disease; Treatment; Rituximab.

\section{INTRODUCTION}

dult-onset Still's disease (AOSD) is a systemic inflammatory disorder of unknown etiology.

Traditional therapies include non-steroidal anti-inflammatory drugs (NSAIDs), corticosteroids, and more recently diseasemodifying anti-rheumatic drugs (1). A number of trials have also been conducted with biological agents [including tumor necrosis factor (TNF) inhibitors, tocilizumab or anakinra], with some promising results (2-4).

We report here the case of a 38-year old female patient with AOSD refractory to cytotoxic agents, successfully treated by repeated rituximab infusion therapy.

\section{CASE REPORT}

A 38-year old female patient without relevant clinical medical history presented with prolonged fever, joint pain, and evanescent rash. The patient's symptoms had begun 4 months before and progressively worsened. She reported high temperature $\left(>39^{\circ} \mathrm{C}\right)$ spiking once a day, usually in the evening or at night, and noticed a salmon pink, maculopapular rash observed with the fever spike localized on the trunk and proximal extremities. She complained of joint pain affecting knees, wrist, and hands. Physical examination showed a $40^{\circ} \mathrm{C}$ fever, confirmed the diffuse rash and revealed bilateral cervical lymph node enlargement. Joint examination confirmed swollen arthritis and a marked tenderness at right wrist radial movement and on squeeze across the metacarpophalangeal joints. Basic laboratory test results revealed marked leukocytosis $\left(17,900\right.$ elements $/ \mathrm{mm}^{3} ; 82 \%$ neutrophils), elevated $\mathrm{C}$ reactive protein of $88 \mathrm{mg} / \mathrm{L}$, hyperfibrinemia of $5 \mathrm{~g} / \mathrm{L}$, hyper $\alpha$ 2 globulinemia of $10 \mathrm{~g} / \mathrm{L}$ on serum electrophoresis and elevated erythrocyte sedimentation rate (ESR) $(70 \mathrm{~mm})$. Ferritin level was also high at $1433 \mathrm{ng} / \mathrm{mL}$. Infectious investigations were negative. Antinuclear antibodies, rheumatoid factors were also negative and complement dosage was normal. Chest X-ray and echocardiography were normal. X-rays of affected joints were normal. Histopathological examination of cervical lymph node biopsy demonstrated polymorphonuclear leukocytes associated
Corresponding author Nabil Belfeki

Department of Internal Medicine

University Hospital of La Rabta

Rue Jebel Lakhdar, 1002, Tunis, Tunisia

E-mail: belfeki.nabil@gmail.com 


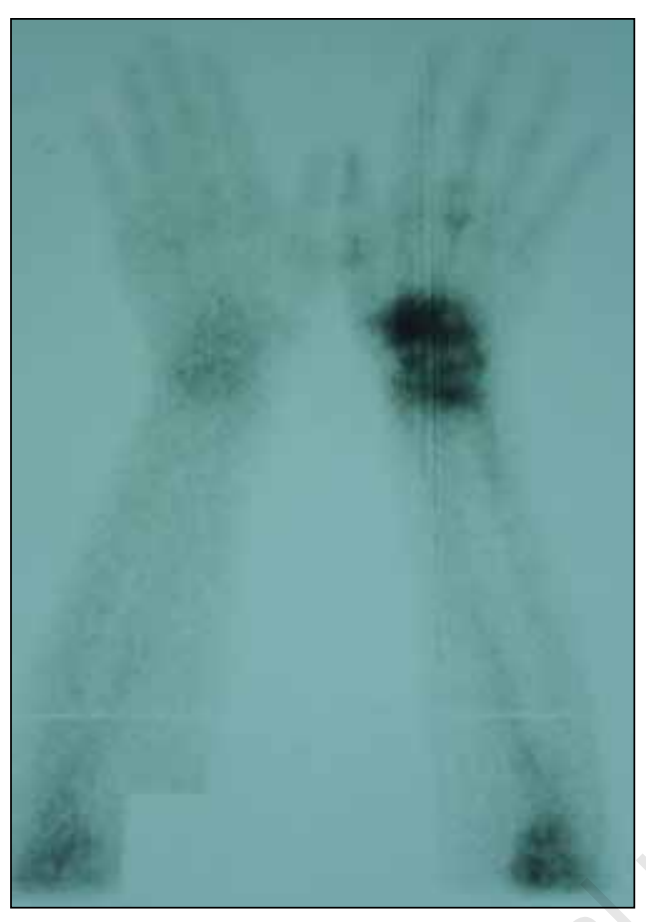

Figure 1 - 99mTC-MDP bone scan delayed planar image of the hands increased tracer uptake within right wrist and carpus.

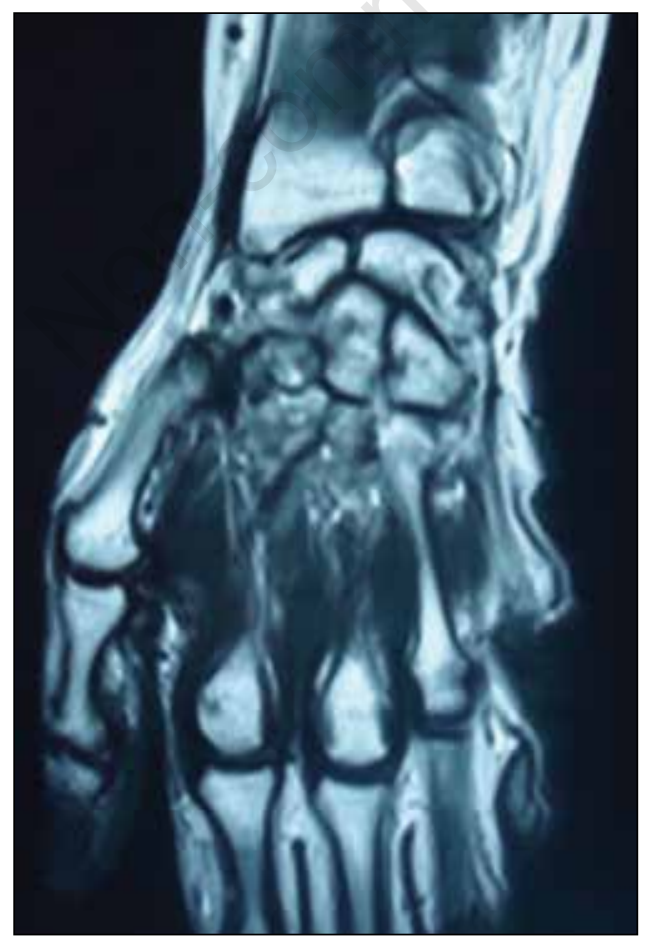

Figure 2 - Wrist magnetic resonance image showing active synovitis with a diffuse carpal arthritis. with signs of reactive hyperplasia. Adult onset Still's disease was diagnosed according to the classification criteria of Yamaguchi et al. and Fautrel et al. $(5,6)$. The patient underwent a course of $60 \mathrm{mg} /$ day of prednisone with progressive tapering, associated with $20 \mathrm{mg}$ /week of methotrexate. The patient remained active 12 months after diagnosis with recurrent febrile episodes, fatigue, right wrist synovitis, and elevated inflammatory markers [mean C-reactive protein (CRP) level of $40 \mathrm{mg} / \mathrm{L}$ and elevated ESR of $45 \mathrm{~mm}$ at the $\left.1^{\text {st }} \mathrm{h}\right]$. The average dosage of prednisone required was $15 \mathrm{mg} /$ day. Radiography of the right wrist showed periarticular osteopenia, radiocarpal and carpometacarpal joints narrowing. 99mTC-MDP bone scan delayed planar image of the hands demonstrated increased tracer uptake within right radio-carpal, radio-ulnar, and intercarpal joints, revealing right wrist arthritis (Figure 1). Right wrist magnetic resonance image showed an active synovitis with an erosive diffuse carpal arthritis (Figure 2) and histopathological examination of synovium biopsy revealed non-specific synovitis. Two intravenous rituximab infusions ( $1 \mathrm{~g}$ ) at 2-week intervals induced remission of wrist pain, fever and skin rash. Inflammatory biomarkers were normal. The concomitant oral steroid dose was decreased from $20 \mathrm{mg}$ to $5 \mathrm{mg}$ oral prednisone/day and methotrexate was continued. The current follow-up from the last rituximab infusion is 12 months without relapsing.

\section{DISCUSSION}

AOSD is a rare systemic inflammatory disorder of unknown origin typically appearing with highspiking fever, a characteristic evanescent, salmon-pink, cutaneous rash and arthritis affecting both small and large joints (7). The treatment of AOSD is often difficult. NSAIDs, steroids, and disease modifying antirheumatic drugs have been shown to be effective in the treatment of AOSD (8). In our case, the major symptoms were oligoarthritis, fever, and skin rash. Despite treatment with prednisone and methotrexate, the patient remained 
active with recurrent febrile episodes, rash, synovitis, and positive inflammatory biomarkers. Although use of methrotrexate is well known to control both chronic systemic symptoms and arthritis, and as a steroid-sparing agent, our patient remained active and over time complicated with wrist erosive arthritis. Two rituximab infusions at 2-week intervals induced remission of synovitis and other symptoms. To our best knowledge, this is the fourth case published on successful treatment of refectory AOSD with rituximab.

Ahmadi et al reported two AOSD patients treated successively with prednisolone methotrexate, ciclosporin, leflunomide, cyclophosphamide per os, and intravenous immunoglobulin. The patients remained active, leading to the use of the TNF inhibitors etanercept and infliximab in one patient and etanercept in the other in combination with methotrexate for an adequate time (8). Despite these different treatments, the patients remained non-responders. Rituximab infusions $\left(375 \mathrm{mg} / \mathrm{m}^{2} \mathrm{IV}\right)$ at 4 -week intervals induced remission of polyarthritis and other symptoms (8). Bartoloni et al. described a 59-year old Caucasian man referred with a history of AOSD diagnosed in 1999.

Methotrexate (MTX) was introduced to avoid chronic use of high doses of CS. Despite adequate treatment, the disease remained active with recurrent febrile episodes, large joint inflammatory involvement, persistent leukocytosis and high ferritin and CRP levels. Use of infliximab $5 \mathrm{mg} / \mathrm{kg}$ in combination with MTX was ineffective. Subsequent introduction of etanercept, $50 \mathrm{mg}$ weekly in combination with MTX, resulted in only transient improvement of disease activity. The drug was discontinued after 18 months because of an adverse event. The introduction of rituximab $1 \mathrm{~g}$ (two infusions at 2-week interval) in combination with MTX induced progressive fever disappearance and polyarthritis regression (9). Rituximab is not a traditional therapy in AOSD. Several studies have suggested that the pathophysiology of AOSD involves the stimulation of lymphoid systems to the point of progression towards lymphoma. Rituximab is a potential treatment option for refractory AOSD (10). In our case, the patient presented with chronic reactive lymph node enlargement, which improved after treatment with rituximab. This leads us to suppose that B cell might represent another target of successful treatment in AOSD. In our case study, we have started with rituximab instead of antiTNF, anti-interleukin (IL)-1, or anti-IL-6 agents. Anti-TNF $\alpha$ seems to be effective in chronic arthritis in AOSD and less effective on systemic symptoms. Resistance to anti-TNF $\alpha$ is not rare and authors suggest that anti-IL- 1 or anti-IL-6 are an interesting alternative in the management of refractory AOSD (11).

Anakinra, an interleukin-1 receptor antagonist, has demonstrated efficacy in single cases and small series of AOSD. However, anakinra seems to be less effective in the joint manifestations than the systemic manifestations. Moreover, serious complications such septicemia and heart failure with anakinra have been reported (12).

Besides, tocilizumab, an IL-6 receptor antagonist, seems to be efficacious in treating adult patients with refractory Still's disease. None of these patients was biologicnaive. Randomized controlled studies are needed to validate these findings (13).

\section{CONCLUSIONS}

The originality of these reports lay in the successful induction of remission with rituximab in biologic-naive AOSD patients. Rituximab should be considered in the treatment algorithm and further studies are needed to determine the place of B cell depletion in AOSD.

\section{REFERENCES}

1. Kadar J, Petrovicz E. Adult-onset Still's disease. Best Pract Res Clin Rheumatol. 2004; 18: 663-76.

2. Efthimiou P, Paik PK, Bielory L. Diagnosis and management of adult onset Still's disease. Ann Rheum Dis. 2006; 65: 564-72.

3. Kobayashi D, Ito S, Murasawa A. Two cases of adult-onset Still's disease treated with tocilizumab that achieved tocilizumab-free remission. Intern Med. 2015; 54: 2675-9. 
4. Ortiz-Sanjuán F, Blanco R, Riancho-Zarrabeitia L. Efficacy of anakinra in refractory adultonset Still's disease: multicenter study of 41 patients and literature review. Medicine (Baltimora). 2015; 94: e1554.

5. Yamaguchi M, Ohta A, Tsunematsu T. Preliminary criteria for classification of adult Stil's disease. J Rheumatol. 1992; 19: 424-30.

6. Fautrel B, Zing E, Golmard JL. Proposal for a new set of classification criteria for adult onset still disease. Medicine. 2002; 81: 194-200.

7. Kontzias A, Efthimiou P. Adult-onset Still's disease: pathogenesis, clinical manifestations and therapeutic advances. Drugs. 2008; 68: 319-37.

8. Ahmadi-Simab K, Lamprecht P, Jankowiak C, Gross WL. Successful treatment of refractory adult onset Stil's disease with rituximab. Ann Rheum Dis. 2006; 65: 1117-8.

9. Bartoloni E, Alunno A, Luccioli F, Santoboni G.
Successful treatment of refractory adult-onset Still's disease with anti-CD20 monoclonal antibody. Clin Exp Rheumatol. 2009; 27: 888-9.

10. Arkfeld DG. The potential utility of B celldirected biologic therapy in autoimmune diseases. Rheumatol Int. 2008; 28: 205-15.

11. Aarntzen EH, Van Riel PL, Barrera P. Refractory adult onset Still's disease and hypersensitivity to non-steroidal anti-inflammatory drugs and cyclo-oxygenase- 2 inhibitors: are biological agents the solution? Ann Rheum Dis. 2005; 64: 1523-24.

12. Ortiz-Sanjuán F, Blanco R, Riancho-Zarrabeitia L. Efficacy of anakinra in refractory adultonset Still's disease: multicenter study of 41 patients and literature review. Medicine (Baltimora). 2015; 94: e1554.

13. Elkayam O, Jiries N, Dranitzki Z. Tocilizumab in adult-onset Still's disease: the Israeli experience. J Rheumatol. 2014; 41: 244-7. 